\title{
Haga usted el diagnóstico Primera parte
}

\author{
Edgar A. Parra ${ }^{1}$, Gustavo Carvajal ${ }^{2}$, Yeni Peña ${ }^{2}$, Jairo Lizarazo ${ }^{2}$ \\ ${ }^{1}$ Grupo de Patología, Instituto Nacional de Salud, Bogotá D.C., Colombia. \\ ${ }^{2}$ Hospital Universitario Erasmo Meoz, Cúcuta, Colombia.
}

Se trata de una mujer de 27 años de edad, procedente de Tibú, Norte de Santander, de ocupación hogar que consultó por cuadro clínico de 8 días de evolución caracterizado por dolor en epigastrio, sensación de masa abdominal, deposiciones melénicas y fiebre no cuantificada.

En el examen físico se encontró a una mujer pálida y desnutrida, febril (temperatura $39^{\circ} \mathrm{C}$ ), con hepatoesplenomegalia, dolor a la palpación en el epigastrio y edemas de los miembros inferiores. Los exámenes de laboratorio mostraron cuadro hemático con hemoglobina de 5,1 g/100, leucocitos de 2.200 por $\mathrm{mm}^{3}$, linfocitos 700 por $\mathrm{mm}^{3}$ y plaquetas 244.000 por $\mathrm{mm}^{3}$; los eritrocitos eran microcíticos e hipocrómicos; la glucemia y la creatinina séricas eran normales y se registraba un leve aumento de las bilirrubinas séricas a expensas de la directa. La hemoclasificación era O positivo, y la prueba de ELISA fue reactiva para VIH.

La ecografía abdominal reveló hepatoesplenomegalia difusa y engrosamiento de las paredes de la vesícula biliar. La TAC de abdomen total mostró múltiples adenomegalias retroperitoneales y mesentéricas, ascitis, hepatoesplenomegalia, edema de la vesícula biliar y derrame pleural derecho (figura 1). La colonoscopia reveló en el recto sigmoides una pequeña erosión de $5 \mathrm{~mm}$ de diámetro, deprimida en el centro y con hematina en su superficie; en el colon transverso

Correspondencia:

Edgar A. Parra S.

Grupo de Patología, Instituto Nacional de Salud, Avenida Calle 26 No. 51-60 Bogotá D.C.

Teléfono: 2207700, extensión 451, 452.

eparra@ins.gov.co.

Recibido: 24/10/05; aceptado: 09/11/05

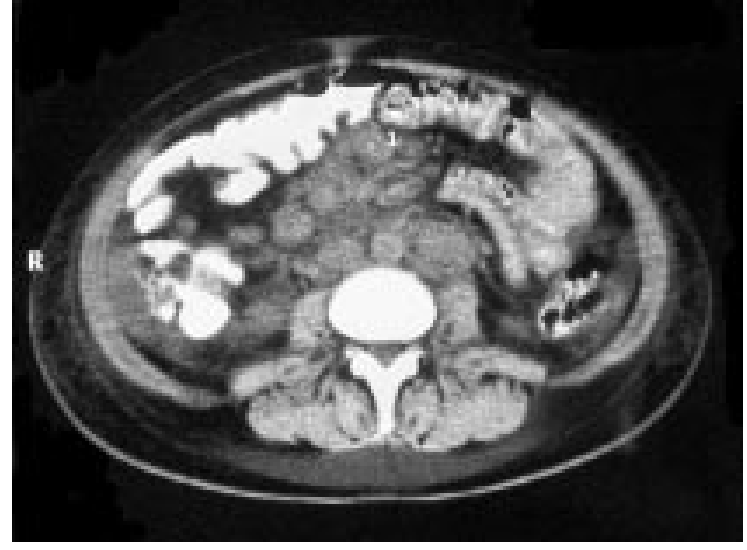

Figura 1. TAC de abdomen que revela múltiples adenopatías mesentéricas y retroperitoneales.

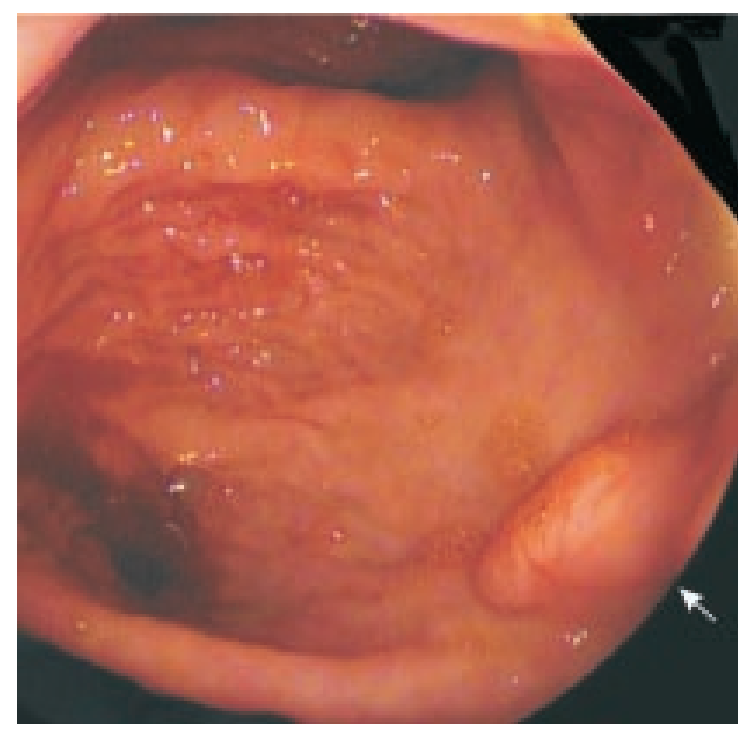

Figura 2. En la colonoscopia se observa una lesión sésil, blanquecina, de unos $6 \mathrm{~mm}$ de longitud, en la mucosa del ángulo hepático. 
medio se identificó una lesión ulcerada nodular de aspecto en sacabocado de $2 \mathrm{~cm}$ de extensión. En el ángulo hepático se encontró en la mucosa una lesión sésil blanquecina de $6 \mathrm{~mm}$, la cual se resecó (figura 2). Se tomaron biopsias en

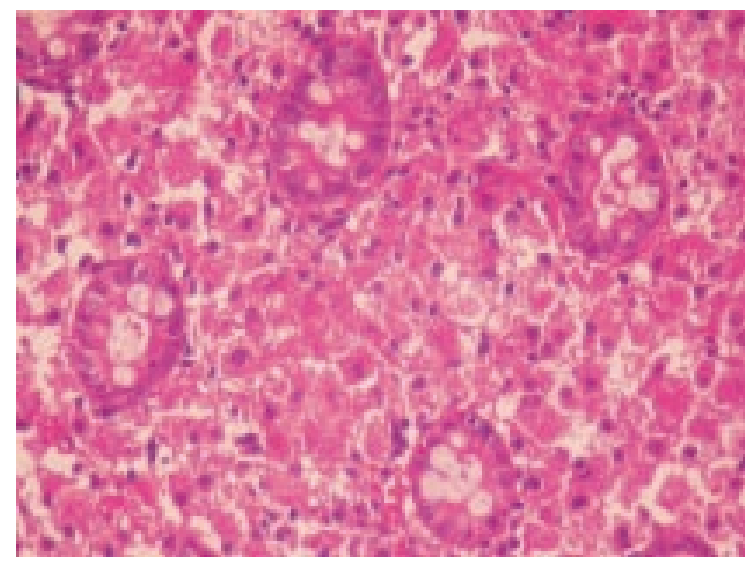

Figura 3. Mucosa de colon, HE, 20X. diferentes localizaciones del colon (figuras 3 y 4 ). El colon ascendente y el ciego no mostraron alteraciones. La paciente falleció 26 días después del ingreso. No recibió tratamiento antirretroviral. ¿Cuál es su diagnóstico?

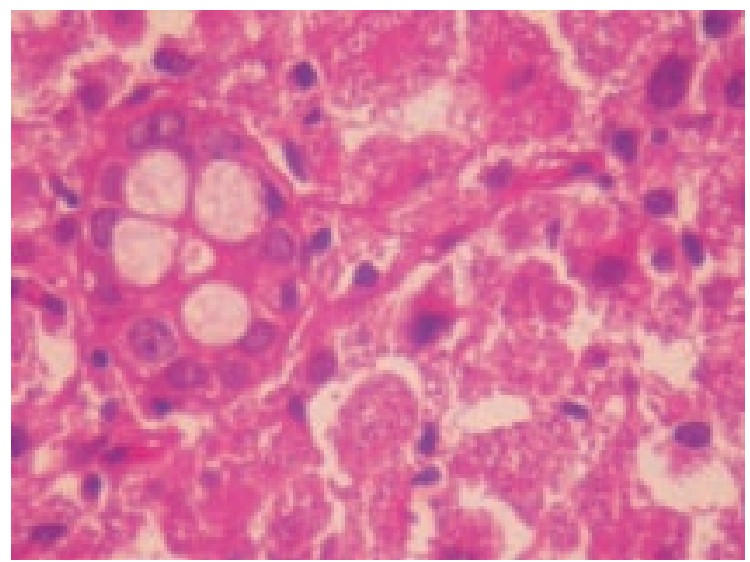

Figura 4. Mucosa de colon, HE, 40X. 


\section{EDUCACIÓN CONTINUA}

\section{Haga usted el diagnóstico Segunda parte}

Edgar A. Parra ${ }^{1}$, Gustavo Carvajal ${ }^{2}$, Yeni Peña ${ }^{2}$, Jairo Lizarazo ${ }^{2}$

${ }^{1}$ Grupo de Patología, Instituto Nacional de Salud, Bogotá, D.C., Colombia.

${ }^{2}$ Hospital Universitario Erasmo Meoz, Cúcuta, Colombia.

El diagnóstico histopatológico fue de histoplasmosis diseminada

En las biopsias de mucosa del colon se identificó infiltrado inflamatorio difuso en la lámina propia; en mayor aumento se observaron pequeñas levaduras esféricas y ovoides que medían 1 a 3 $\mu \mathrm{m}$ de diámetro. Estos microorganismos se visualizaron con la coloración de plata metenamina; el estudio inmunohistoquímico fue positivo para Histoplasma capsulatum (figuras 5 a 8$)$.

La histoplasmosis es una micosis sistémica relativamente común que causa morbilidad en pacientes residentes en áreas endémicas y que ha emergido como importante complicación de los pacientes con infección por el VIH $(1,2)$. La enfermedad es causada por el hongo dimórfico H. capsulatum var. capsulatum y fue descrita por primera vez en 1905 por Samuel Darling; en Colombia, el primer caso fue informado en 1947 por Gast Galvis (3). La micosis se adquiere por inhalación de las partículas infectantes (microconidias, restos de micelio) producidas por el hongo en su hábitat natural y en el suelo, especialmente si está contaminado con excrementos de aves y murciélagos. La enfermedad es altamente endémica en ciertas regiones de Norteamérica y se la encuentra también, aunque en menor proporción, en Centro

\footnotetext{
Correspondencia:

Edgar A. Parra

Grupo de Patología, Instituto Nacional de Salud, Avenida Calle 26 No. 51-60 Bogotá D.C.

Teléfono: 220 7700, extensión 451, 452.

eparra@ins.gov.co.
}

Recibido: 24/10/05; aceptado: 09/11/05 y Suramérica; no obstante, se la ha informado en otras partes del mundo; la infección primaria ocurre en el pulmón y suele cursar asintomática en $90 \%$

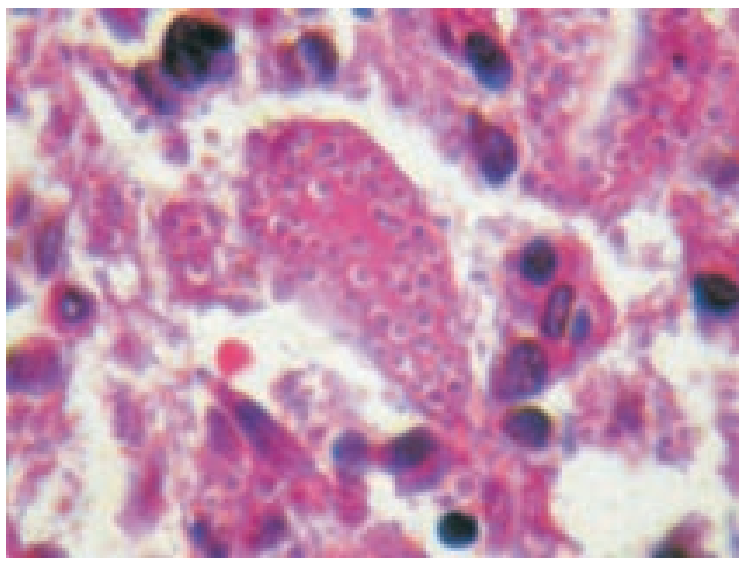

Figura 5. HE, 40X. En la lámina propia de la mucosa del colon se observan numerosos histiocitos con abundantes levaduras intracelulares que miden de 1 a 3 micras de diámetro.

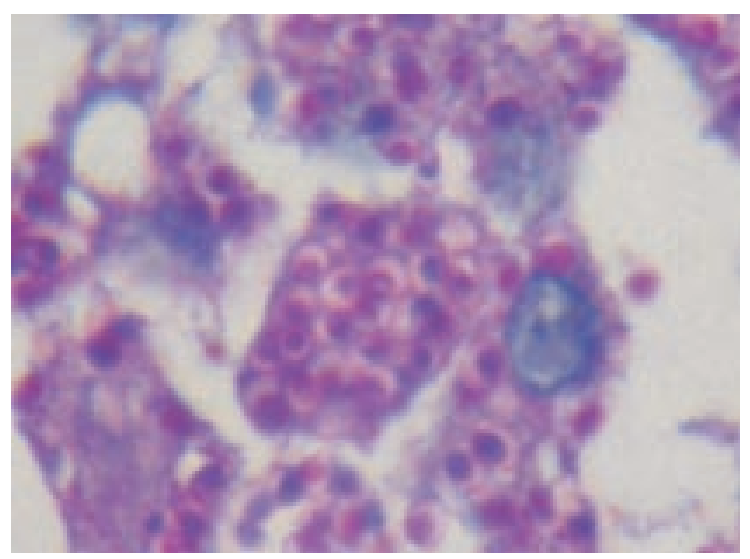

Figura 6. PAS, $60 \mathrm{X}$. Las levaduras son de forma redondeada y ovoide, se identifica un halo claro perinuclear que genera la falsa imagen de cápsula sin teñir. 


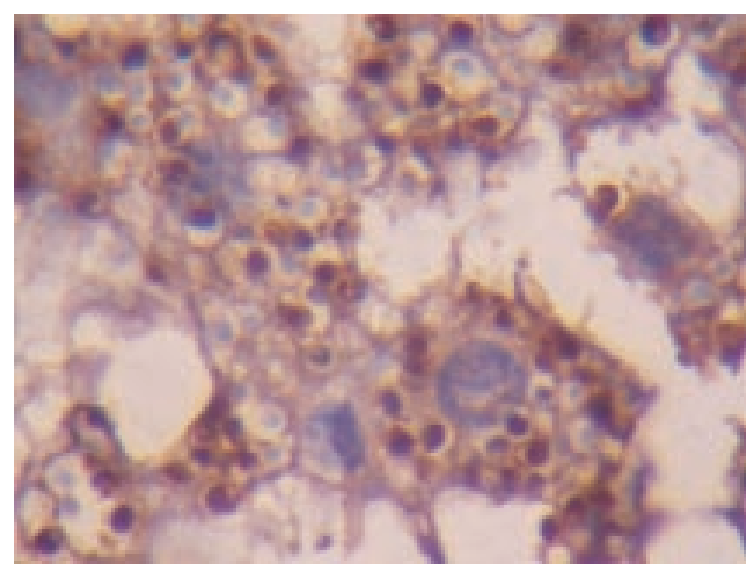

Figura 7. Inmunorreactividad positiva para histoplasma, técnica de avidina-biotina-peroxidasa, 40X.

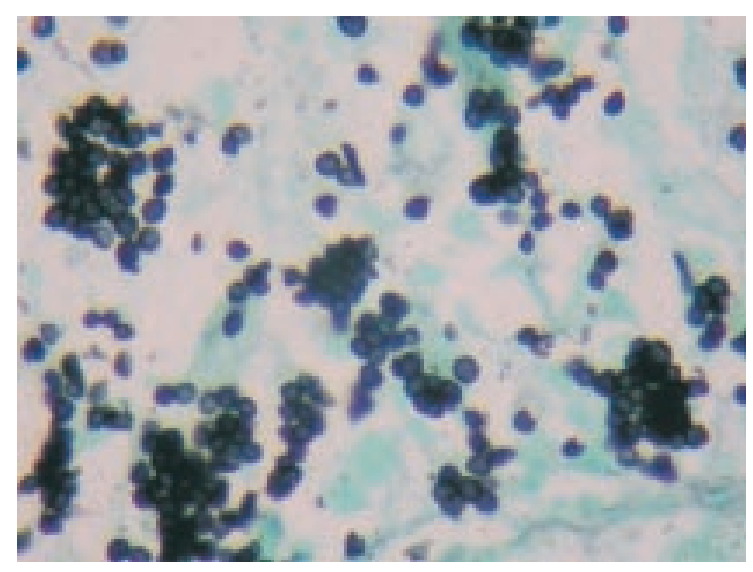

Figura 8. Plata metenamina-Gomori, 40X. Las coloraciones argénticas permiten visualizar los detalles morfológicos de las levaduras, así como confirmar el diagnóstico en los diferentes tipos de micosis sistémicas que afectan al huésped.

a 95\% de los casos; clínicamente la enfermedad se presenta en varias formas, a saber, pulmonar aguda, pulmonar crónica y diseminada. El periodo de incubación varía entre 3 y 14 días según la gravedad de la respuesta inmune del hospedero y del número de partículas infecciosas inhaladas $(2,4)$.

En Colombia, la reactividad a la histoplasmina informada en algunas áreas endémicas señala que hasta una tercera parte de los adultos jóvenes han estado en contacto con el hongo (5).

La histoplasmosis diseminada es la forma más grave de la enfermedad, usualmente ocurre en edad avanzada o en individuos con estados de inmunodeficiencia. Se presenta como complicación de las neoplasias hematológicas, la quimioterapia y la terapia inmunosupresora en pacientes con trasplante y sida. La diseminación hematógena resulta de la infección progresiva de varios órganos por la vía del sistema mononuclear fagocítico siendo la mortalidad cercana a $80 \%$ sin terapia específica (6).

La histoplasmosis primaria gastrointestinal no es común y en la mayoría de las circunstancias se pesenta en el contexto de una enfermedad diseminada en hospederos inmunosuprimidos. En el sida, este tipo de histoplasmosis es una complicación poco frecuente, pero que suele observarse en áreas endémicas. El íleon y la región cecal son las localizaciones más frecuentes; las lesiones se caracterizan por ser pseudopolipoides, ulceradas, pseudotumorales o con engrosamiento de la pared intestinal; clínicamente, se puede presentar hemorragia intestinal, dolor abdominal, obstrucción y malabsorción; la perforación y peritonitis son frecuentes en el intestino delgado (7). En algunas ocasiones se puede confundir con cáncer de colon, especialmente en pacientes inmunocompetentes $(8,9)$ y paracoccidioidomicosis $(10)$.

\section{Conflicto de intereses}

Los autores declaran que no existe conflicto de intereses en esta publicación.

\section{Financiación}

Para la realización de este trabajo, los autores no han contado con fuente alguna de financiación externa.

\section{Referencias}

1. Arango M, de Bedout C, Tobón AM, Restrepo A, Torrado E, Castañeda $\mathrm{E}$ et al. Histoplasmosis en Colombia: estudio interinstitucional. Inf Quinc Epidemiol Nac 2000;5:119-23.

2. Deepe GS Jr. Histoplasmosis capsulatum. En: Mandell GL, Bennett JE, Dolin R, eds. Principles and practice of infectious disease. 5th ed. Philadelphia: Churchill Livingstone; 2000; p.2718-33.

3. Gast Galvis A. Histoplasmosis en Colombia. Anales de la Sociedad de Biología de Bogotá 1947;2:203-7.

4. Tobón A, Franco L. Histoplasmosis en el adulto. Acta Med Col 1997;22:277-84. 
5. Restrepo A, Robledo M. Distribution of histoplasmina sensitivity in Colombia. Am J Trop Med Hyg 1968;17: 25-37.

6. Connor DH, Chandler FW. Histoplamosis capsulati. In: Pathology of infectious diseases. Stamford, Conn: Appleton \& Lange; 1997. p.1017-22.

7. Jani JC, Brown R, Kajdacsy-Balla A, Guzmán G. Pathologic quiz case. A woman with human immunodeficiency virus with right lower quadrant pain and ascending colon mass. Arch Pathol Lab Med 2005; 129:259-61.

8. Lee JT, Dixon MR, Murrell Z, Konyalian V, Agbunag R, Rostami S et al. Colonic histoplasmosis presenting as colon cancer in the non-immunocompromised patient: report of a case and review of the literature. Am Surg 2004;70:959-63.

9. Goulet CJ, Moseley RH, Tonnerre C, Sandhu IS, Saint S. Clinical problem-solving. The unturned stone. N Eng J Med 2005;352:489-94.

10. Bedoya AM, Vélez A, Tobón AM, Juliao F, Ruiz M, Arango $\mathbf{M}$ et al. Paracoccidioidomicosis diseminada tipo juvenil con compromiso intestinal. Presentación de un caso. Rev Colombiana Gastroentorol 2002;17: 252-5. 